\title{
Lack of association between fibromyalgia and sleep apnoea syndrome
}

\author{
Bonifacio Alvarez Lario, Joaquin Teran, Jose L Alonso, Javier Alegre, Ignacio Arroyo, \\ Jose L Viejo
}

factor, antinuclear antibodies and osteoarticular radiography were carried out when necessary.

To determine whether sleep disorders can cause a fibromyalgia syndrome, 30 patients with sleep apnoea syndrome were studied. All presented an important reduction in deep sleep stages (-93.1 (SD 17.9)\% of stage IV and $-77 \cdot 2(45 \cdot 7) \%$ of stage III) and frequent episodes of wakening ('arousals'), factors which are involved in fibromyalgia. One patient (3\%) met the criteria for fibromyalgia; the estimated prevalence of fibromyalgia for patients attending a general medical clinic is $6 \%$. No significant correlation was found between the number of points which were tender upon pressure and the various sleep parameters studied. It is concluded that sleep disorders alone are not able to produce a fibromyalgia syndrome.

The origin of fibromyalgia syndrome is not well known. Several theories about the aetiopathogenesis of fibromyalgia syndrome have been proposed, but none can adequately explain it. ${ }^{1}$ Clinical symptoms of sleep anomalies are frequently noted in patients with fibromyalgia syndrome, ${ }^{2}{ }^{3}$ and sleep electroencephalographic studies of these patients have shown quantitative and qualitative disorders in non-rapid eye movement sleep. ${ }^{4}$ These were the first physiopathological changes described in this disorder. The provocation of fibromyalgia syndrome in normal subjects deprived of sleep stage IV $^{5}$ and the clinical response of some patients with fibromyalgia syndrome to drugs to increase deep sleep ${ }^{6}$ support the proposal that sleep disorders may be of pathogenic importance to fibromyalgia syndrome.

If this proposal is valid, it would be expected that other disorders which are related to frequently interrupted sleep, such as sleep apnoea syndrome, should be strongly related to fibromyalgia syndrome.

\section{Patients and methods}

Thirty consecutive patients with sleep apnoea syndrome examined during routine clinic checks were studied between September 1988 and February 1990. All patients were examined physically by a pneumologist and a rheumatologist and the haemogram, erythrocyte sedimentation rate (Westergren), overall biochemical profile, total proteins and electrophoretic pattern and thyroid hormones were determined. Pulmonary function testing, a chest radiogram and an electrocardiogram were performed. Other tests such as for rheumatoid

Correspondence to: Dr Bonifacio Alvarez Lario, Sección de Reumatología, Hospital General Yaguie, Avda. del Cid Campeador $s / n$ 09005, Burgos, Spain.

Accepted for publication 8 January 1991

\section{CRITERIA FOR SLEEP APNOEA SYNDROME}

Sleep apnoea syndrome was defined as the presence of at least 10 apnoeas per sleep hour which appeared during the rapid eye movement and non-rapid eye movement stages. An apnoea was defined as a cessation of air flow for at least 10 seconds. $^{78}$ The apnoea index was determined as the total number of apnoeas divided by the number of sleep hours.

\section{CRITERIA FOR FIBROMYALGIA SYNDROME}

The patients were diagnosed as having fibromyalgia syndrome when they reported pain in at least three anatomical sites for more than three months. ${ }^{9}$ It was essential that the patient felt pain when applying manual pressure in at least seven of the 14 painful points specified by Smythe (intertransverse $\mathrm{C} 4-\mathrm{C} 6$, midpoint upper trapezius, medial border of scapula, second costochondral joint, L4-S1 interspinous ligament, mid gluteus, $2 \mathrm{~cm}$ distal to lateral epicondyle and medial fat pad proximal to knee). ${ }^{10}$ The patients' response to such tender points being touched was rated as severe, moderate, mild, or none. Any indication of pain ('mild or greater') was accepted as a tender point. Though not essential for the diagnosis, the patients were asked about the modulating factors considered as minor criteria by Yunus $e t$ $a l^{3}$ and the fibromyalgic symptoms were quantified by the Campbell questionnaire for fibromyalgia syndrome screening in a general population.

\section{POLYSOMNOGRAPHIC STUDY}

The parameters monitored by polysomnography included electroencephalography (F3/C4, F4/C4, C3/A2, C4/A1), nasal air flow (by a thermoflux nasal respiration transducer), eye movement (by electro-oculography), submental electromyography, abdominal and thoracic respiratory effort (by the thoracic-abdominal band), electrocardiography, and haemoglobin oxygen saturation (by cutaneous pulse oximetry). The conventional parameters of sleep stages were studied using the electroencephalography criteria described by Reschtschaffen and Kales. ${ }^{11}$

\section{STATISTICAL STUDY}

The Spearman coefficient was used to correlate quantitative variables. The Mann-Whitney U 
Selected characteristics of the 30 patients with sleep apnoea syndrome studied in this work

\begin{tabular}{lr}
\hline Characteristics & Mean value (SD) \\
\hline Age (years) & $57 \cdot 1(7 \cdot 7)$ \\
Number of tender points & $2 \cdot 2(2 \cdot 3)$ \\
Number of modulating factors & $1 \cdot 2(1 \cdot 2)$ \\
Reduction in stage III sleep (\%) & $-77 \cdot 2(45 \cdot 7)$ \\
Reduction in stage IV sleep (\%) & $-93 \cdot 1(17 \cdot 9)$ \\
Apnoea per hour index & $30 \cdot 3(12 \cdot 8)$ \\
\hline
\end{tabular}

test was used to compare the means. A two tailed test was performed in all instances. Values of $p \leqslant 0.05$ were considered as statistically significant.

\section{Results}

The main findings for the patients included in the study are shown in the table. The average age was $57 \cdot 1$ years (range 38-70). Three of the 30 patients were women. The average number of 'mild or greater' tender points was $2 \cdot 2(2 \cdot 3)$. The average number of moderate and severe tender points was $1 \cdot 3(1 \cdot 7)$. The average number of modulating factors was $1 \cdot 2(1 \cdot 2)$, poor sleep $(43 \%)$, chronic headache $(37 \%)$ and tiredness ( $27 \%$ ) being the most frequent.

Only one patient $(3 \%)$ met the proposed criteria for fibromyalgia syndrome. Two patients reported widespread pain (at least three anatomical sites): the patient diagnosed as having fibromyalgia syndrome and a patient diagnosed with rheumatoid arthritis. The Campbell questionnaire for possible fibromyalgia syndrome was positive in two patients, i.e. the patient meeting the criteria for fibromyalgia syndrome and another patient who did not show tender points on manual examination and suffered from unspecified chronic low back pain. Following the Yunus criteria, which require modulating factors, only the former patient met the criteria for fibromyalgia syndrome.

All patients presented an important reduction on the percentage of non-rapid eye movement sleep stage IV $(-93 \cdot 1(17 \cdot 9) \%$ over the percentage of theoretical time) and non-rapid eye movement sleep stage III $(-77 \cdot 2(45 \cdot 7) \%$ over the percentage of theoretical time) (table and fig 1). Surprisingly, and despite the important sleep alterations, 17 patients $(57 \%)$ insisted that they slept well at night and 20 patients $(67 \%)$ said they felt refreshed the following morning.

All patients were more than $20 \%$ overweight compared with the ideal weight for their age and

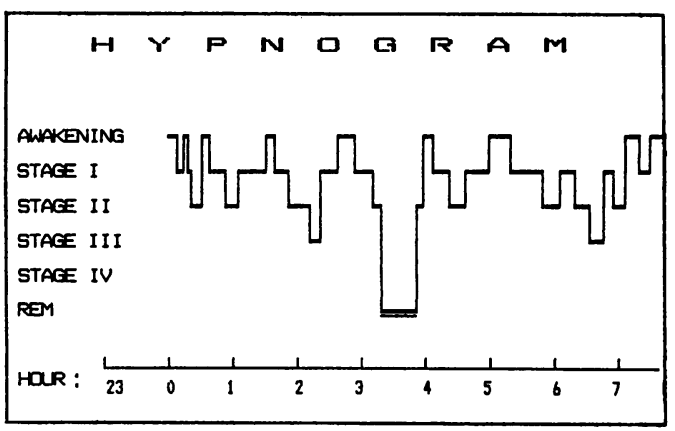

Figure 1 Hypnogram of a patient with sleep apnoea syndrome. A reduction can be seen in sleep stages $I I I$ and $I V$, as well as an important fragmentation of sleep. height according to standard tables. No patient practised sports on either a regular or sporadic basis.

All patients presented more than 10 apnoeas per sleep hour, as required by the criteria for sleep apnoea syndrome. The apnoea index was $30.3(12 \cdot 8)$ apnoeas per sleep hour. All patients showed a decrease of at least $5 \%$ in the oxygen saturation level during the apnoeas; the mean minimum oxygen saturation was $72 \cdot 5(7 \cdot 6) \%$.

No significant correlation was found between either the number of tender points and the reduction in non-rapid eye movement sleep at stages III and IV, nor between the number of points painful to pressure and the apnoea index. No significant differences were found between the mean number of points painful to pressure in patients who said that they slept well and the mean number of tender points in patients who said they slept badly. This result was also found when comparing patients with a subjective feeling of having slept well with those who slept badly.

Apart from the patient with fibromyalgia syndrome, the following rheumatological diagnoses were made: osteoarthritis in five patients (cervical spine in two, lumbar spine in two, knees in two, and hand in two patients), gouty arthritis in two patients and asymptomatic hyperuricaemia in another three patients, unspecified chronic low back pain in one patient, rheumatoid arthritis in one patient and shoulder rotator cuff tendinitis in one patient. This frequency was considered normal for the age and characteristics of the patients studied.

\section{Discussion}

There are no generally accepted criteria for the diagnosis of fibromyalgia syndrome. The most frequently used criteria are the presence of aching in three anatomical locations lasting more than three months, as well as pain on manual pressure in at least seven specific tender points. ${ }^{9}$ The Campbell questionnaire was suggested for the study of possible fibrositis on an overall population, the presence of multiple tender points being necessary for a confirmatory diagnosis. The Yunus criteria ${ }^{3}$ require a lower number of tender points, but the presence of modulating factors is necessary. The same controversy arises as for the number and location of the different tender points required for diagnosis, though several workers have suggested seven as an adequate minimum number. ${ }^{9}{ }^{12}$ It has been noted that the clinical features of patients with fibromyalgia syndrome diagnosed using the three different sets of criteria are uniform. ${ }^{6}$ In the same way, the only patient diagnosed with fibromyalgia syndrome in our study met the criteria of all three sets of features. A unified criterion for the diagnosis of fibromyalgia syndrome $e^{13}$ was published after this work had been performed.

The aetiology and pathogenesis of fibromyalgia syndrome are unknown. The physiopathological mechanisms most frequently mentioned are the presence of local muscular lesions, immune alterations, neurotransmitter imbalance, psychiatric alterations and, sleep disorders. 


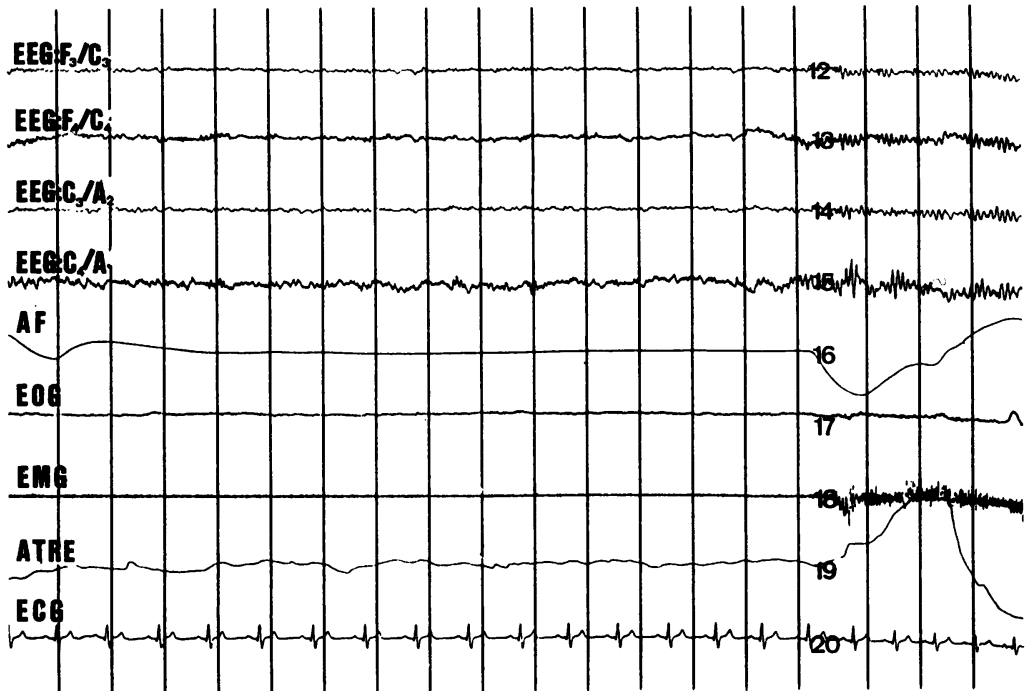

Figure 2 Polysomnographic study of a patient with sleep apnoea syndrome in whom apnoea can be observed followed by arousal and an alpha rhythm in electroencephalography. Abbreviations: $E E G=$ electroencephalogram; $A F=$ air flow; $E O G=$ electro-oculogram; $E M G=$ electromyogram; $A T R E=a b d o m i n a l$ and thoracic respiratory effort; $E C G=$ electrocardiogram.
Molony et al, of whom only one patient met the criteria for fibromyalgia syndrome following either one of the above mentioned criteria. All our patients led a sedentary life. Therefore it was hardly possible to assume that practising sports 'protected' them from fibromyalgia syndrome. Although the prevalence of fibromyalgia syndrome has not been thoroughly established, it is believed that about $6 \%$ of the population attending a general medical clinic and about $20 \%$ of patients referred to an academic rheumatology clinic meet the criteria for fibromyalgia syndrome. ${ }^{2}{ }^{3}$ In our series, no increase in the frequency of fibromyalgia syndrome is found in patients with sleep apnoea syndrome. Therefore the high frequency found by Molony et al ${ }^{17}$ could be attributed to the limited size and bias of their sampling; only 11 of 32 patients with sleep apnoea syndrome consented to clinical rheumatological evaluation, probably those with more musculoskeletal disorders.

As the apnoea index might be considered as an indirect measurement of the number of endogenous wakenings (most apnoeas, although not all, are followed by a partial or total wakening) (fig 2), we tried to correlate the number of tender points on pressure and the apnoea index, but no significant correlation was found. In a similar way no correlation was found between the decreasing percentage of non-rapid eye movement sleep stages III and IV and the presence of a greater or smaller number of tender points and/or the number of modulating factors. No significant differences were observed when comparing the mean numbers of tender points in patients who slept well or badly or rested well or badly.

A total of 27 of our 30 patients were men and could favour the view that men are more resistant than women to the effects of sleep disturbance. Psychological disorders, neurotransmitter imbalance or other factors could also be involved in the origin of fibromyalgia syndrome, the presence of a number of them being necessary for the development of fibromyalgia syndrome.

These findings lead to the conclusion that the deprivation of deep sleep does not seem to be able to produce a fibromyalgia syndrome by itself. Thus the fibromyalgia sleep alterations described are only part of the symptomatic complex (like headache or irritable colon)-that is, the cause of this disease is multifactorial.

We thank Dr Pedro Abaigar for techical assistance. fibromyalgia-like symptoms in healthy subjects deprived of non-rapid eye movement sleep stage IV. 5

Patients with sleep apnoea syndrome present an important reduction of deep sleep stages ${ }^{7}$ (fig 1) as well as numerous episodes of wakening (partial or total arousals), frequently followed by alpha rhythm intrustion in electroencephalography (fig 2). ${ }^{16}$ Therefore sleep apnoea syndrome is a good pattern of the sleep disorders suggested as aetiopathogenic factors in fibromyalgia syndrome. Molony et $\mathrm{al}^{17}$ studied 11 patients with sleep apnoea syndrome, of whom three were found to meet the criteria for fibromyalgia syndrome ( $27 \%)$. We studied 30 patients with sleep apnoea syndrome with criteria for fibromyalgia syndrome similar to those used by
1 Goldenberg D L. Research in fibromyalgia: past, present and future. F R heumatol 1988; 15: 992-6.

2 Campbell S M, Clark S, Tindall E A, Forehand M E, Bennett R M. Clinical characteristics of fibrositis. I. A "blinded" controlled study of symptoms and tender points. Arthritis Rheum 1983; 26: 817-24.

3 Yunus M B, Masi A T, Calabro J J, Miller K A, Feigenbaum S L. Primary fibromyalgia (fibrositis): clinical study of 50 patients with matched normal controls. Semin Arthritis Rheum 1981; 11: 151-71.

4 Moldofsky H, Scarisbrick P, England R, et al. Musculoskeletal symptoms and non-REM sleep disturbance in patients with 'fibrositis syndrome' and healthy subjects. Psychosom Med 1975; 37: 341-51.

5 Moldofsky $\mathrm{H}$, Scarisbrick $\mathrm{P}$. Induction of neurasthenic musculoskeletal pain syndrome by selective sleep stage deprivation. Psychosom Med 1976; 38: 35-44.

6 Moldofsky H, Lue F A. The relationship of alpha and delta 
EEG frequencies to pain and mood in "tibrositis" patients treated with chlorpromazine and L-tryptophan. Electro encephalogr Clin Neurophysiol 1980; 50: 71-80.

7 Shepard J W. Physiologic and clinical consequences of sleep apnea. Seminars in Respiratory Medicine 1988; 9: 560-8.

8 Stradling J R, Phillipson E A. Breathing disorders during sleep. $Q \mathcal{F}$ Med 1986; 58: 3-18.

9 Wolfe F, Cathey M A. Prevalence of primary and secondary fibrositis. F R heumatol 1983; 10: 965-8.

10 Smythe $\mathrm{H}$ A. Non-articular rheumatism and the fibrositis syndrome. In: Hollander J L, McCarthy D J Jr, eds. Arthritis and allied conditions. 8th ed. Philadelphia: Lea \& Febiger, 1972: 874-84.

11 Rechtschaffen A, Kales A, eds. A manual of standardized terminology: techniques and scoring system for sleep stages of terminology: techniques and scorning system for sleep stages of
human subjects. VS Public Health Service, 1968. (NIH publication No 204.)
12 Wolfe F, Hawley D J, Cathey M A, Caro X, Russell I J. Fribrositis: symptom frequency and criteria for diagnosis. f Rheumatol 1985; 12: 1159-63.

13 Wolfe F, Smythe H A, Yunus M B, et al. The American College of Rheumatology 1990 criteria for the classification of fibromyalgia. Report of the multicenter criteria comof fibromyalgia. Report of the multicenter
mittee. Arthritis Rheum 1990; 33: 160-72.

14 Mahowald M W, Mahowald M L, Bundlie S R, Ytterberg $S$ R. Sleep fragmentation in rheumatoid arthritis. Arthritis Rheum 1989; 32: 974-83.

15 Moldofsky H. Sleep and musculoskeletal pain. Am $\mathcal{F}$ Med 1986; 81: 585-9.

16 Smith P L. Evaluation of patients with sleep disorders. Seminars in Respiratory Medicine 1988; 9: 534-9.

17 Molony R R, McPeek D M, Schiffman P L, et al. Sleep, sleep apnea and the fibromyalgia syndrome. 7 Rheumatol 1986; 13: 797-800. 